\title{
Deposition Temperature- and Solvent-Dependent 2D \\ Supramolecular Assemblies of Trimesic Acid at the Liquid-Graphite \\ Interface Revealed by Scanning Tunneling Microscopy
}

\author{
Doan Chau Yen Nguyen ${ }^{1}$, Lars Smykalla ${ }^{1}$, Thi Ngoc Ha Nguyen ${ }^{1}$, Tobias Rüffer ${ }^{2}$, Michael \\ Hietschold $^{1}$ \\ ${ }^{1}$ Technische Universität Chemnitz, Institute of Physics, Solid Surfaces Analysis Group, \\ ${ }^{2}$ Technische Universität Chemnitz, Institute of Chemistry, Inorganic Chemistry, \\ D-09107 Chemnitz, Germany.
}

\section{Supporting Information:}

Figure S1: The series images of evaporation of the solvent are inevitable according to experimental observations.
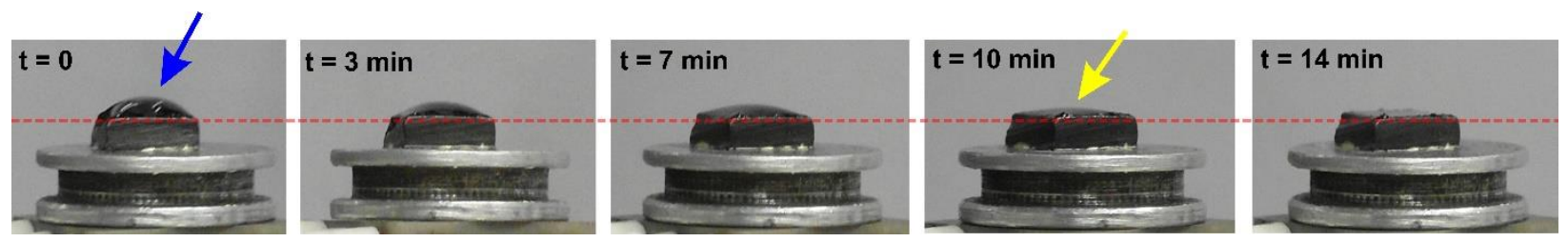

Figure S1: The series images of evaporation of the solvent are inevitable according to our experimental observations. A drop of the TMA/Octanoic acid solution (about $5 \mu \mathrm{L}$ marked by blue arrow) was deposited on the $50^{\circ} \mathrm{C}$ preheated surface of a piece of freshly cleaved HOPG $(5 \times 5 \mathrm{~mm}$, the same piece as used for STM measurement). The solution on the sample was measured both before and after heating for 14 min (marked by a dot red line). This experiment showed that ca. $50 \%$ of the solution evaporated after heating in 10 minutes. Therefore, the TMA concentration in the octanoic droplet on the substrate must increase with time. 